\title{
Unified Description of the Resonance Peak and Incommensuration in High- $T_{c}$ Superconductors
}

\author{
C.D. Batista, G. Ortiz, and A.V. Balatsky \\ Theoretical Division, Los Alamos National Laboratory, Los Alamos, NM 87545
}

(Received November 5, 2018)

\begin{abstract}
We present a unified description of the resonance peak and low-energy incommensurate response observed in high- $T_{c}$ cuprate superconductors. We argue that both features have a purely magnetic origin and they represent universal features of an incommensurate spin state both below and above the superconducting transition temperature. In this description the resonance peak is the reflection of commensurate antiferromagnetism. Our theoretical scenario gives an account of the main features observed in various families of superconductors and predicts those not yet observed, like a resonance peak in $\mathrm{La}_{2} \mathrm{NiO}_{4+x}$.
\end{abstract}

Neutron scattering experiments in the cuprates reveal two interesting and seemingly unrelated effects: (a) the low-energy incommensurate peaks at momenta $\mathbf{k}=$ $\left(\frac{\pi}{a}, \frac{\pi}{a}(1 \pm \delta)\right)$ and $\left(\frac{\pi}{a}(1 \pm \delta), \frac{\pi}{a}\right)$ [1] with incommensuration $\delta$ (in units of $\frac{\pi}{a}$ ) and, (b) the so-called resonance peak at energies close to $40 \mathrm{meV}$ in the spin susceptibility [2] at the antiferromagnetic $(\mathrm{AF})$ vector $\mathbf{Q}=\left(\frac{\pi}{a}, \frac{\pi}{a}\right)$. These experimental observations are widely believed to be important for our understanding of the nature of the magnetic correlations and ultimately for the understanding of the superconductivity in high- $T_{c}$ materials.

On the one hand the intensity and the energy $E_{r}$ of the resonance peak seems to scale with the superconducting coherence energy scale [3]

$$
E_{r} \simeq 5 k_{B} T_{c} .
$$

This experimental observation was in fact used in Refs. [4] to relate the formation of the superconducting coherence to the opening of a new spin-scattering channel in the superconducting state that is impossible in the normal state above the superconducting critical temperature $T_{c}$. Several theoretical scenarios attributed the origin of the resonance peak to superconducting coherence effects [5] or considered it as the fingerprint of a collective mode in an $S O(5)$ symmetric field theory $[6]$.

On the other hand the direct proportionality between $\delta$ and $T_{c}$ has been observed in recent neutron scattering data for LSCO and YBCO compounds [7]

$$
k_{B} T_{c}=2 \hbar v^{*} \delta
$$

with some characteristic and material dependent velocity $\hbar v^{*} \sim 17-35 \mathrm{meV} \AA$, where $\delta$ is measured in units of $\frac{\pi}{a}$.

One could argue that there is no immediate connection between the two phenomena [8]. In this case any relationship to superconductivity is accidental and hence there is no unifying physics to be learned from comparing these two sets of observations. Alternatively one can attempt to prove that these two phenomena are intimately related. This is the point of view we will advocate in this article: We argue for the common origin of both the low energy incommensurate response and resonance peak as a magnetic scattering in the disordered incommensurate spin state. In our interpretation the resonance peak is the spectral weight at energy $E_{r}$ associated to the lowest energy spinon excitation with $\mathbf{k}=\mathbf{Q}$ in a system with spin incommensuration. A key aspect of our analysis is the realization that the experimental observations are different manifestations of a unique physical phenomenon.

It is apparent that charge doping induces a certain spin ordering in these low-dimensional, doped, antiferromagnets [11] as a result of competing interactions. Indeed, in one spatial dimension this can be exactly shown to be the case [9]. Here, we will only consider the spin degrees of freedom and show that, regardless of the nature of the spin correlations, universal features emerge as a result of an arbitrary magnetic incommensuration. A relevant question is, however, up to what degree the charge channel affects the spin response and therefore our conclusions. Since one spatial dimension is the case that can be unambiguously addressed we have performed calculations on the $1 d t-J$ model to show that our main thesis remains unchanged in the presence of charges.

From Eqs. (11) and (2) one can conclude that

$$
E_{r}=\alpha \delta
$$

with $\alpha \simeq(8-10) \hbar v^{*}$. This equation could indeed imply that the resonance peak energy and incommensuration are directly related.

We find that there are direct experimental predictions based upon our analysis that can help to resolve whether the incommensuration and resonance are direct consequences of an incommensurate spin state. These are:

- Goldstone modes, characteristic of an ordered AF state, are shifted away from $\mathbf{Q}$ by $\pm \frac{\pi}{a} \delta$ with a local maximum in the dispersion relation $\omega_{\mathbf{k}}$ at $\mathbf{k}=\mathbf{Q}$.

- $E_{r} \propto \delta$, for small $\delta$.

- Resonance peak is associated with the lowest energy quasiparticle excitation at $\mathbf{k}=\mathbf{Q}$.

- Absolute intensity of the resonance peak decreases when: the spin gap $\Delta_{s}$ (fixed $\delta$ ), or $\delta\left(\right.$ fixed $\Delta_{s}$ ), or the temperature $T$ increases. 
We show that these features (illustrated in Figs. 1 and 2) are universal, independently of the way the incommensuration is established.

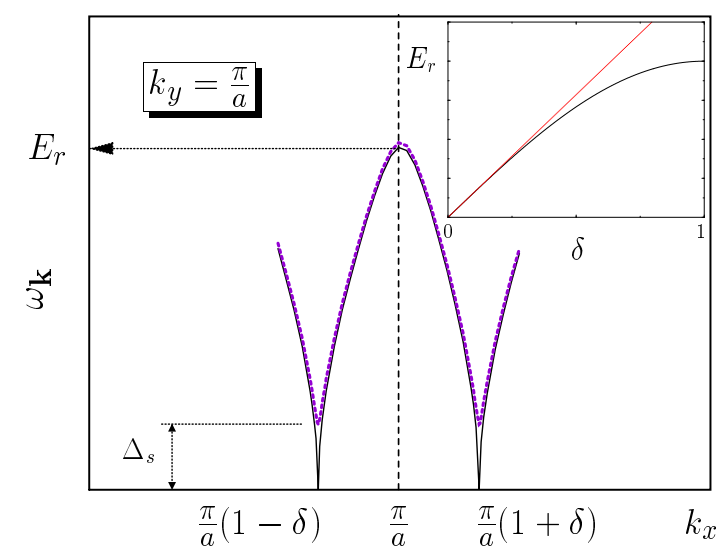

FIG. 1. Spin-wave dispersion relation for an incommensuration in the $x$-direction of $\pm \frac{\pi}{a} \delta$, i.e., $\tilde{\mathbf{Q}}=\frac{\pi}{a}(1 \pm \delta, 1)$. For completeness, we also display the case where there is an spin gap $\Delta_{s}$ in the dispersion relation. For $\Delta_{s}=0$, we recover the massless Goldstone bosons at $\mathbf{k}=\tilde{\mathbf{Q}}$. The departure from linearity of the resonance energy at $\mathbf{Q}$ is shown in the inset.

To support such a claim we now present three different examples of spin incommensuration $\left(\mathbf{S}_{i}^{2}=S(S+1)\right)$ with a common AF background:

(i) The first example corresponds to "striped" topological ordering, where on top of an AF backbone state $|A F\rangle$ there is a "ferromagnetic" (FM) incommensurate exchange coupling $J^{\prime}>0$ that we model as

$$
H^{\prime}=-J^{\prime} \sum_{\langle\alpha, \beta\rangle} \mathbf{S}_{\alpha} \cdot \mathbf{S}_{\beta},
$$

where $\langle\alpha, \beta\rangle$ labels the FM link. The striped arrangement of FM links induces $\pi$-shifted AF domains. A straightforward perturbative argument leads to $E_{r}=$ $\frac{N_{L}}{N} J^{\prime}\left\langle A F\left|\mathbf{S}_{\alpha} \cdot \mathbf{S}_{\beta}\right| A F\right\rangle \propto \delta J^{\prime}$, where the ratio between the number of FM links $N_{L}$ and lattice sites $N$ is $\delta$.

(ii) The second is a spin-wave argument. Assume that on top of an AF background there is a sinusoidally modulated spin structure such that $\left\langle S_{i}^{z}\right\rangle=S \cos \left(\tilde{\mathbf{Q}} \cdot \mathbf{r}_{i}\right)$, with $\tilde{\mathbf{Q}}=\mathbf{Q} \pm\left(\frac{\pi}{a} \delta, 0\right)$. This incommensurate spin structure can be stabilized by adding an adequate term to a Heisenberg Hamiltonian [12]. When one considers the simplest AF Hamiltonian the spin propagator $G_{i j}(t)=$ $-i\left\langle\hat{T} S_{i}^{+}(t) S_{j}^{-}(0)\right\rangle$ has an equation of motion

$$
\begin{aligned}
{\left[\omega^{2}+\omega_{0}^{2}\right] } & G\left(\mathbf{k}, \mathbf{k}^{\prime} ; \omega\right)=\omega S\left[\delta_{\mathbf{k}, \mathbf{k}^{\prime}+\tilde{\mathbf{Q}}}+\delta_{\left.\mathbf{k}, \mathbf{k}^{\prime}-\tilde{\mathbf{Q}}\right]}\right. \\
& +f(\mathbf{k}-\tilde{\mathbf{Q}}) f(\mathbf{k}-2 \tilde{\mathbf{Q}}) G\left(\mathbf{k}-2 \tilde{\mathbf{Q}}, \mathbf{k}^{\prime} ; \omega\right) \\
& +f(\mathbf{k}+\tilde{\mathbf{Q}}) f(\mathbf{k}+2 \tilde{\mathbf{Q}}) G\left(\mathbf{k}+2 \tilde{\mathbf{Q}}, \mathbf{k}^{\prime} ; \omega\right)
\end{aligned}
$$

with momentum-dependent frequency $\omega_{0}^{2}=-f(\mathbf{k})[f(\mathbf{k}+$ $\tilde{\mathbf{Q}})+f(\mathbf{k}-\tilde{\mathbf{Q}})]$ and $f(\mathbf{k})=S[-J(\tilde{\mathbf{Q}})+J(\mathbf{k})]$, where $J(\mathbf{k})$ is the Fourier transform of the exchange interaction. This can be easily solved by using either a continued fraction representation 12 of $G$ or mapping its equation of motion to a Harper-like Hamiltonian [13] that can be diagonalized using standard methods. A simple analysis shows that for small $\delta, E_{r} \propto \delta$ while Goldstone modes appear at $\tilde{\mathbf{Q}}$. The spin-wave dispersion relation $\omega_{\mathbf{k}}$ has a local maximum at $\mathbf{k}=\mathbf{Q}$ (see Fig. 11).

(iii) Finally, one can use a Schwinger boson mean-field description of an AF Hamiltonian, as in the spin-wave case, including a term which gives rise to an incommensurate spin phase [14]. Even though the Schwinger-boson approach does not explicitely break the spin $S U(2)$ symmetry, as in the spin-wave approximation, both give the same qualitative features for $\omega_{\mathbf{k}}$ and the dynamic magnetic structure factor $S(\mathbf{k}, \omega)$.

From these examples one recognizes that certain features are universal, regardless of the way the incommensuration is established. In Fig. 11 we show the low-energy spin-wave dispersion relation obtained following examples (ii) and (iii) which basically lead to the same qualitative results. We observe the appearance of Goldstone modes at the incommensurate wave vector $\tilde{\mathbf{Q}}$. The resonance energy $E_{r}$ (inset) is linear in $\delta$ for the values of incommensuration observed experimentally. We have also considered the case where there is a spin gap $\Delta_{s}$ in the excitation spectrum 15. Note that the value of $E_{r}$ is not greatly affected by the introduction of a spin gap.

At low energies $S(\mathbf{k}, \omega)$ provides information on the spectral weight of the lowest energy spin-quasiparticle excitations (Fig 2). In the presence of long range order, Goldstone bosons appear at $\mathbf{k}=\tilde{\mathbf{Q}}$ in $\omega=0$ due to the spontaneously broken $S U(2)$ symmetry. The spectral weight, that is proportional to the magnetic susceptibility $\chi(\mathbf{k}, \omega)$, then diverges at $\mathbf{k}=\tilde{\mathbf{Q}}$ and $\omega \rightarrow 0$. In the presence of a spin gap (as it seems to be the case for the cuprates) these peaks become finite and indicate the presence of short-range spin correlation at $\mathbf{k}=\tilde{\mathbf{Q}}$.

The low-energy peaks merge into a broad commensurate response at $\mathbf{k}=\mathbf{Q}$ (Fig. 2), where the resonance peak emerges as a fingerprint of the incommensurate nature of the spin state. The intensity of the resonance peak decreases with increasing $\delta$. This can be understood as follows: The incommensurate phase is an AF state modulated with a small $\delta$; therefore, in the presence of incommensurability, there are still AF regions of size $\frac{2 a}{\delta}$ in the $x$ direction. Under these conditions we expect a high intensity for the spectral weight of the lowest energy spin excitation at $\mathbf{k}=\mathbf{Q}$ which is reminiscent of the divergent weight which is obtained when the $\mathbf{k}=\mathbf{Q}$ channel is gapless and the size of the AF region is infinite. In the lower panel of Fig. 2 2 we clearly show that both the low-energy incommensurate response $(S(\mathbf{k}, \omega)$ for fixed energy $\left.\omega_{0}\right)$ and the resonance peak $(S(\mathbf{k}, \omega)$ at 
$\mathbf{k}=\mathbf{Q}$ ) have the same magnetic origin.

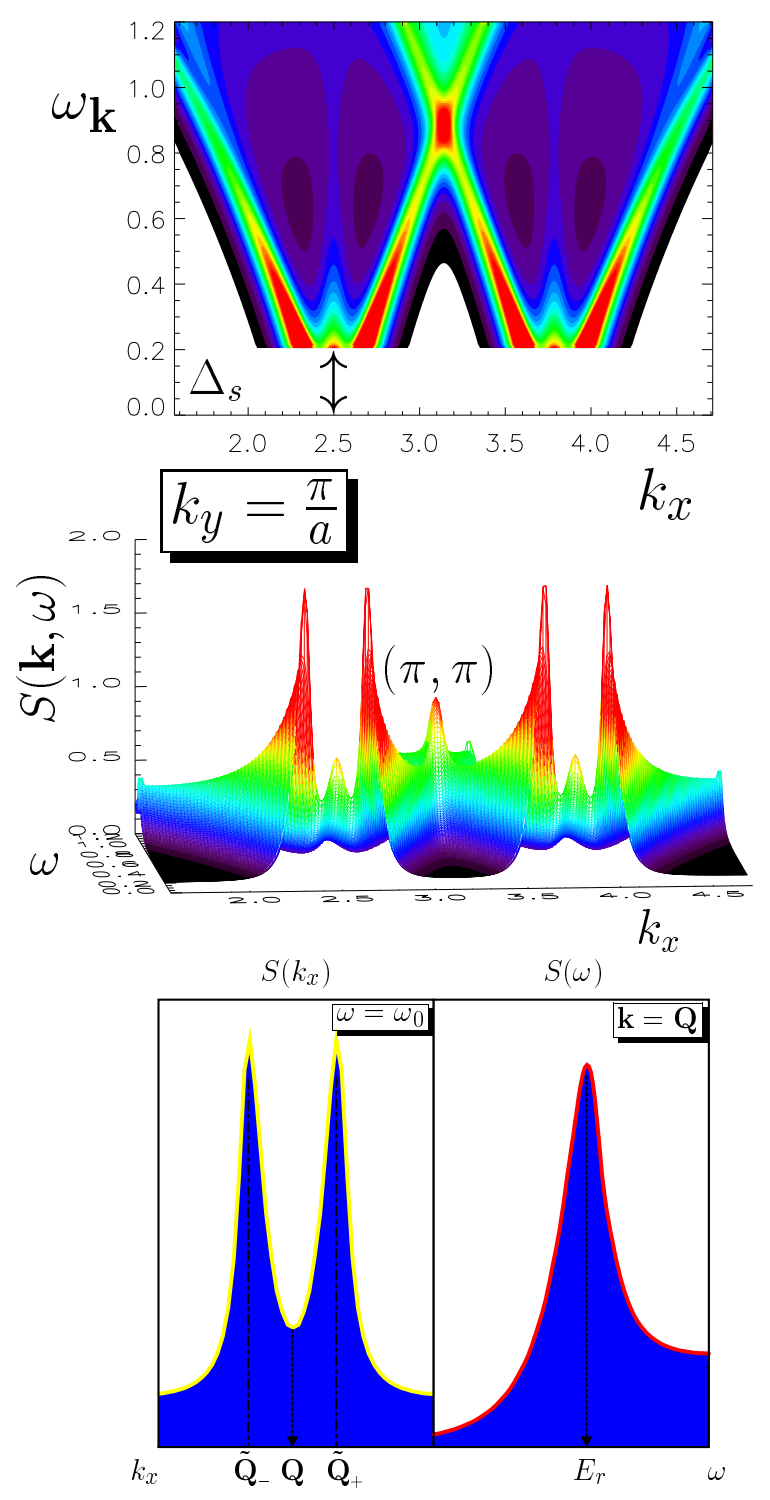

FIG. 2. Energy and momentum dependence of the incommensurate spin fluctuations as computed from case (iii). The upper panel is a contour plot of the lower one that clearly indicates the existence of a resonance peak at $\mathbf{k}=\mathbf{Q}$. The intensity diminishes as one moves away from the AF wave vector $\mathbf{Q}$. The modes at $\mathbf{k}=\tilde{\mathbf{Q}}_{ \pm}$and $\omega \rightarrow 0$ are strongly affected by the spin gap $\Delta_{s}$. As $\Delta_{s}$ increases there is a relative increase of weight at the $(\pi, \pi)$-resonance. The lower panel displays two cuts of the function $S(\mathbf{k}, \omega)$, one in momentum and the other in energy $\left(\omega_{0}<E_{r}\right)$. The incommensurate vectors are $\tilde{\mathbf{Q}}_{ \pm}=\mathbf{Q} \pm \frac{\pi}{a}(\delta, 0)$.

We consider now the effect the charge degrees of freedom have on the spin dynamics. To this end, we have calculated $S(\mathbf{k}, \omega)$ for a $1 d t-J$ model (16 sites); the results are shown in Fig. 3. Clearly, the main universal features are still there: Goldstone modes at the incommensurate points, $(\pi, \pi)$ peak at $\mathbf{k}=\mathbf{Q}$, etc. The particle chan- nel contributes to establish the incommensuration (each charge carries an anti-phase boundary for the AF order parameter [9]) but once it is established the main qualitative features in the spin dynamics remain unchanged. This seems to be the case for any doped AF Mott insulator whenever its state is spin incommensurate.

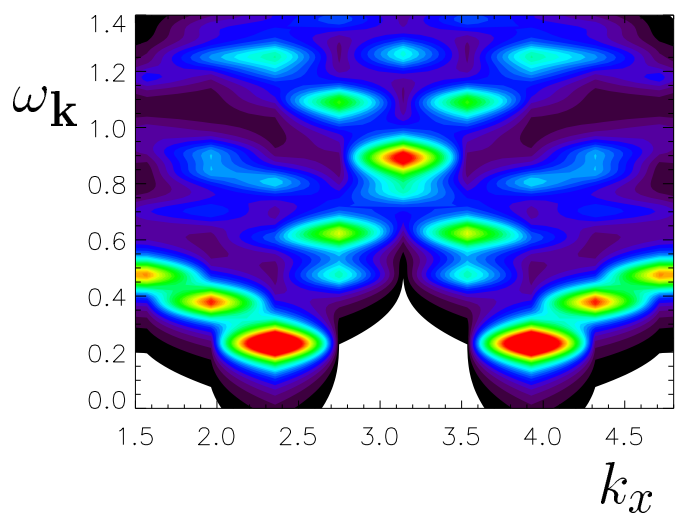

FIG. 3. Same as upper panel in Fig. 2 but for a model with both charge and spin degrees of freedom. The hole concentration is $n_{h}=\frac{1}{4}$ and $J / t=0.4$.

These generic features have been observed in YBCO and Bi2212. In LSCO the incommensuration has been certainly measured although there is no indication of the presence of a resonance peak. However, this question still has to be clarified experimentally, although it is possible that a great degree of disorder in this compound is responsible for washing out the peak and decrease its intensity. Our calculation indicates that the relative weight of the resonance peak decreases and the weight at the incommensurate points increases with decreasing spin gap $\Delta_{s}$. This might explain why the resonance has not been observed in LSCO where the spin gap, $\Delta_{s} \sim 7 \mathrm{meV}$, is substantially smaller than in YBCO. From our picture we predict a resonance peak at $E_{r}=14-16 \mathrm{meV}$ for LSCO. Table If summarizes the magnetic properties of the different families of cuprate superconductors. Using the linearity between $T_{c}$ and $\delta$, and the fact that $E_{r}$ is linear in $\delta$ for an incommensurate system (with small incommensuration $\delta$ ), we can also explain the linear relation between $E_{r}$ and $T_{c}$ measured in YBCO and Bi2212. Note that our interpretation provides a definite meaning to the velocity $\hbar v^{*}$, which is directly proportional to the rate of change of the resonance energy $E_{r}$ as the result of changes in the spin incommensuration $\delta$.

Within our magnetic scenario one can qualitatively understand why in the superconducting phase the intensity of the $(\pi, \pi)$ peak will be enhanced, independently of the mechanism that drives the superconducting state: Since holes moving in an uncorrelated fashion in an incommensurate AF background carry an anti-phase domain wall, whenever superconducting or pairing fluctuations coexist they will tend to "annihilate" those domains (holes form 
pairs) and make the system more AF. These fluctuations produce some degree of disorder with wavelengths longer than the incommensurate wavelength. The net effect is a transfer of intensity in $S(\mathbf{k}, \omega)$ from the $\mathbf{k}$-points near the incommensurate wavevectors $\tilde{\mathbf{Q}}_{ \pm}$towards the $\mathbf{k}$-points near the commensurate point $\mathbf{Q}$ (this is seen in calculations of the $1 d t-J$ model). This scenario is consistent with the temperature dependence of the incommensurate magnetic response observed in $\mathrm{La}_{1.86} \mathrm{Sr}_{0.14} \mathrm{CuO}_{4}$ [8].

To sharpen our argument about the purely magnetic origin of the resonance peak in the incommensurate state we turn now to the non-superconducting $\mathrm{La}_{2} \mathrm{NiO}_{4+x}$. This is a material where the existence of charged stripes is well established [18]. From our model we expect that in the striped phase of $\mathrm{LaNiO}$ the magnetic incommensuration coexists with the resonance peak. We estimate $E_{r} \sim 40-70 \mathrm{meV}$ for $\mathrm{La}_{2} \mathrm{NiO}_{4.13}$. It would be interesting to see whether high-energy neutron scattering experiments can be done on this material to search for a resonance peak in an insulating material.

In conclusion, we argued that both the low energy incommensurate response and resonance peak have a common magnetic origin. They are natural consequences of an incommensurate spin state. Superconductivity is not the cause of the resonance peak although it could be the consequence of incommensuration. There is an anomaly in the temperature dependence of the resonance peak at $T=T_{c}$ with an abrupt change in intensity above the level of a weaker, normal response. From our description this fact can be related to the decreasing number of anti-phase boundaries due to pairing fluctuations. These fluctuations transfer spectral weight from the region around the incommensurate $\mathbf{k}$-points into the region near the commensurate $\mathbf{k}=\mathbf{Q}$ point [8]. In addition, the presence of a gap, regardless of the superconducting mechanism, reduces the spin-particle scattering increasing the antiferromagnetic fluctuations. $E_{r}$ emerges as the result of the competition between antiferromagnetism (which can lead to charge confinement) and delocalization (driven by kinetic energy) therefore defining an additional characteristic energy scale. From our findings, $E_{r}$ is intimately related to the emergent length scale $\delta$. The purely magnetic origin of the resonance peak explains why it has been observed above $T_{c}$. Most of the previous descriptions of the origin of the $(\pi, \pi)$-resonance [5, 4, 6] invoked coherent effects or collective modes present in the superconducting state. These theories cannot explain the many cases where the resonance has been observed above the superconducting transition [8]. An indirect confirmation of our phenomenology is related to recent experimental observations on the effect of nonmagnetic Zn impurities in YBCO [19]. It is known that Zn-doping suppresses superconductivity and at the same time leads to a resonance peak above $T_{c}$, seemingly due to increased $\mathrm{AF}$ correlations.

Work at Los Alamos is sponsored by the US DOE un- der contract W-7405-ENG-36. We also acknowledge useful discussions with P. Bourges, P.C. Dai, I. Martin, H.A. Mook, D.K. Morr, J.M. Tranquada, and S. Trugman.

[1] J.M. Tranquada et al., Nature 375, 561 (1995).

[2] J. Rossat-Mignod et al., Physica B 192, 109 (1993); J. Rossat-Mignod et al., in Selected Topics in Superconductivity Frontiers in Solid State Sciences Vol 1., eds. L.C. Gupta and M.S. Multani (World Scientific 1993), 265.

[3] P. Bourges, in The gap Symmetry and Fluctuations in High Temperature Superconductors, eds. J. Bok, G. Deutscher, D. Pavuna, and S.A. Wolf. (Plenum Press, 1998); L.P. Regnault et al., in Neutron Scattering in Layered Copper-Oxide Superconductors, ed. A. Furrer (Kluwer, Amsterdam, 1998).

[4] I.I. Mazin and V. Yakovenko, Phys. Rev. Lett. 75, 4134 (1995); E. Demler and S. -C. Zhang, Phys. Rev. Lett. 75, 4126 (1995); D. Z. Liu, Y. Zha, and K. Levin, Phys. Rev. Lett. 75, 4130 (1995); N. Bulut and D. J. Scalapino, Phys. Rev. B 53, 5149 (1996); B. Normand, H. Kohno and H. Fukuyama, J. Phys. Jpn. 64, 3903 (1995).

[5] J.P. Lu, Phys. Rev. Lett. 68, 125 (1992); Y. Ohashi and H. Shiba, J. Phys. Soc. Jap. 62, 2783 (1993); P. Monthoux and D.J. Scalapino, Phys. Rev. Lett. 72, 2131 (1994); M. Lavagna and G. Stemmann, Phys. Rev. B 49, 4235 (1994); F. Onufrieva and J.M. Rossat-Mignod, Phys. Rev. B 52, 7572 (1995); F. Onufrieva and P. Pfeuty, cond-mat/9903097; D.K. Morr and D. Pines, Phys. Rev. Lett. 81, 1086 (1998).

[6] S-C. Zhang, Science 275, 1089 (1997).

[7] K. Yamada et al., Phys. Rev. B 57, 6165 (1998); A.V. Balatsky and P. Bourges, Phys. Rev. Lett. 82, 5337 (1999); M. Arai et.al., cond-mat/9912233; H.A. Mook et al., Nature 395, 580 (1998). See also ISIS report at http://www.isis.rl.ac.uk/ISIS98/feat11.htm; A.V. Balatsky and Z.X. Shen, Science 284, 1137 (1999).

[8] T.E. Mason, in Handbook on the Physics and Chemistry of Rare Earths, eds. K.A. Gschneidner Jr., L. Eyring, and M.B. Maple.

[9] C.D. Batista and G. Ortiz, Phys. Rev. Lett. 85, 4755 (2000).

[10] P. Bourges et al., Science 288, 1234 (2000).

[11] Bilayer materials like YBCO introduce another higher energy scale in the problem leading to optical branches in the spin excitation spectrum that we are not going to consider in our analysis.

[12] S.H. Liu, J. of Magn. Magn. Mater. 22, 93 (1980).

[13] T. Ziman and P-A. Lindgard, Phys. Rev. B 33, 1976 (1986).

[14] B. Normand and P.A. Lee, Phys. Rev. B 51, 15519 (1995).

[15] The spin gap is an intrinsic function of $\delta$ in the real compounds.

[16] H. Mook et al., cond-mat/9811100.

[17] H. He et al., cond-mat/0002013; H. Fong et al., Nature 
398, 588 (1999).

[18] J.M. Tranquada, P. Wochner, and D.J. Buttrey, Phys. Rev. Lett. 79, 2133 (1997).

[19] Y. Sidis et al., cond-mat/0006265.

\begin{tabular}{lcccc}
\hline$\hbar v^{*}(\mathrm{meV} \AA)$ & 17 & 35 & 35 & 29 \\
\hline$E_{r}(\mathrm{meV})$ & $\mathbf{1 4 - 1 6}$ & 41 & 34 & 37 \\
\hline$\delta$ (r.l.u.) & 0.10 & 0.12 & 0.11 & 0.16 \\
\hline$T_{c}(K)$ & 30 & 89 & 62.7 & 84 \\
\hline Refs. & 0 & $\mathbf{1 0}, 0]$ & 0 & 16 \\
\hline
\end{tabular}

TABLE I. Properties of various families of high- $T_{c}$ compounds. Note that $E_{r}=14-16 \mathrm{meV}$ is our prediction for $\mathrm{LS}_{0.1} \mathrm{CO}$ based upon the linear relation between $E_{r}$ and $\delta$. Precise values for $\hbar v^{*}, \delta$ and $E_{r}$ are taken from experimental data. The proportionality $E_{r} \sim T_{c} \sim \delta$ holds within $10 \%$

\begin{tabular}{llllll}
\hline Compound & $\mathrm{LS}_{0.1} \mathrm{CO}$ & $\mathrm{YBCO}_{6.85}$ & $\mathrm{YBCO}_{6.6}$ & $\mathrm{Bi} 2212$ & accuracy. \\
\hline
\end{tabular} 